\title{
Feel \& Look Beautiful Through Ayurveda!
}

\section{Neelam $P^{*}$ \\ Department of preventive Cardiology \& Diabetes, India}

*Corresponding author: Patil Neelam, Ayurvedic Consultant, Panchkarma Therapy, Gurukrupa NR Satidevi mandir, Eksar village Borivili, west Mumbai, India, Tel: 9892518107; Email: neelampatil74@gmail.com

\section{Clinical Note}

Volume 2 Issue 2

Received Date: May 08, 2018

Published Date: May 11, 2018

\section{Clinical Note}

\section{Skin}

This is the largest organ in our body, which is in direct contact with the outside world. Hence skin to be protected from birth itself. Indians practice of anointing the skin with Sandalwood and turmeric. On all religions purpose to enhance beauty. Modern stress of the working class, excessive unprotected sun exposure are all contributing to an early loss of inherent beauty of the skin.

You are unique and so is your skin. So first step is to identify your skin.

Skin Type - For example whether your skin is Oily, Dry, sensitive. It needs to be cleansed regularly and treated accordingly.

\section{Recommended Diet for Healthy Skin}

- Eat a balanced diet that includes vegetables, fruits, grains, nuts.

- Consume a protein rich diet such as Meat, Fish, and Eggs. For vegetarians they may consider soya , nuts , legumes

- Have juice made with tomato, beetroot, and spinach daily.

- Eat seasonal fruits daily.

- Have 1 cup of curd regularly.

- Drink plenty of water to promote hydration in skin.

- Avoid foods high in sugar.

- Avoid a high carbohydrate diet i.e. limit your intake of white rice, pasta, potatoes, sweets
- Avoid fried and highly seasoned foods.

\section{Skin Care}

The skin needs basic and natural treatment for itself, most of the face washes, soaps, face creams contain chemicals which affect sensitive skin. Hence one must use natural homemade skin care routines.

\section{Lemon}

Lemon is the Most Popular Source of Vitamin C and is a Must Have in almost Every Diet because of its Amazing Health and Skin Benefits.

\section{Applications}

- Squeeze Lemon Juice In to Half a Cup of Warm Water and Drink it first thing in the Morning

- Lemon Juice Mixed With Cold Water Makes a Great Refreshing Drink.

- Lemon Juice Mixed With Ginger Juice Gives our Body Cleansing Effects.

- Cut The Lemon in half, Store this in a Glass Bottle Mixed with $100 \mathrm{~mol}$ of Water and refrigerate it. You Can Use this as a Conditioner as Final Hair wash, and Drink it to Clean Your Body.

- Take 2 Tsp Lemon Water, Mix it With Milk Cream and Use it as a Moisturizer for Dry Skin.

\section{Sugar}

- Powdered sugar when mixed with Honey, peppermint essence, coconut oil can work as a natural face scrub. 


\section{Journal of Natural \& Ayurvedic Medicine}

- Another way to use powdered sugar is to mix brown sugar and grinded coffee with coconut oil which will help detoxify the skin.

- The mixture of powdered sugar, vanilla essence, coconut or jasmine oil, one spoon of honey as per your needs can be used as body scrub and it will help moisturize your skin.

\section{Sandal wood}

- Sandalwood can act as a good face brightener, Mix powered sandalwood with some water and apply it on your face. Let the mixture dry for 2-3 minutes and wash it off. It leaves a cool and refreshing effect on ones skin. 